\title{
SIMPLE KELVIN EQUATION APPLICABLE IN THE CRITICAL POINT VICINITY
}

\author{
Valeev A.A.
}

Kamyshin Technological Institute of Volgograd State Technical University, Kamyshin, e-mail:waleev@mail.ru

\footnotetext{
New simple form of the Kelvin equation is offered that applies near the gas-liquid transition critical point.
}

Keywords: Kelvin equation, critical point, phase transition, nano-structured materials

Well known form of the Kelvin Equation [1, 2]

$$
\frac{\tilde{p}_{s a t}}{p_{s a t}}=\exp \left(-\frac{\mu}{\rho_{L} R T} \frac{2 \sigma}{r}\right)
$$

has been proved to work excellent for the phase equilibrium of rare gas and liquid systems, the ones it was originally designed for. Here $p_{\text {sat }}$, $\tilde{p}_{\text {sat }}$ are the saturated vapor pressures against a plain surface and a meniscus with effective curvature radius $r$, respectively, the latter accounting for such effects as the adsorption at the pore wall. Number " 2 " stays for spherical geometry of the meniscus. It should be replaced by " 1 " in the case of the cylindrical geometry. $\mu$ is the molar mass of the substance in use, $\rho_{\text {r }}$ is the liquid phase density, $R$ is the universal gas constant. Quite often, this equation is misused when applied to high-presser gases and to liquids at near-critical temperatures.

To account for the gas's nonideality, another equation [3] was developed:

$$
\frac{\tilde{f}_{\text {sat }}}{f_{\text {sat }}}=\exp \left(-\frac{\mu}{\rho_{L} R T} \frac{2 \sigma}{r}\right)
$$

where $f$ is the replacement of the pressure by the fugacity. In the next paragraph, it will be shown that this equation, as well as those obtained in [4], does not work quite well near the critical point and the replacement will be presented.

\section{Kelvin equation from scratch}

We consider a liquid-gas equilibrium system divided by a plain surface. Now we can put down the change in the Gibbs energy for each phase:

$$
d G_{i}=d \mu_{i} n_{i}=-S_{i} d T_{i}+V_{i} d p_{i}+\mu_{i} d n_{i},
$$

where $i$ denotes the phase that can be either $G$ or $L . \mu_{i}, n_{i}, S_{i}, T_{i}, V_{i}, p_{i}$ are chemical potential, amount of substance, entropy, temperature, volume and pressure of the phase $i$, respectively. Taking into account that both phases are in the state of thermodynamical equilibrium, we can put:

$$
T_{G}=T_{L} \equiv T, \quad \mu_{G}=\mu_{L} \equiv \mu .
$$

Generally speaking, $p_{G} \neq p_{L}$, due to additional pressure by the curved surface tension. The eq. (3) will take the following form:

$$
d G_{i}=d \mu n_{i}=-S_{i} d T+V_{i} d p_{i}+\mu d n_{i} .
$$

Now we can put down the change in the chemical potential:

$$
d \mu=d\left(G_{i} / n_{i}\right)=-s_{i} d T+v_{i} d p_{i},
$$

where $s_{i}, v_{i}$, are the molar entropy and the molar volume, respectively. Now we can see that

$$
(d \mu)_{T, n_{i}}=v_{i} d p_{i} .
$$

Taking into account that $p_{L}=p_{G}-2 \sigma / r$, we can put

$$
(d \mu)_{T, n_{i}}=v_{G} d p_{G}=v_{L} d\left(p_{G}-2 \sigma / r\right) .
$$

Omitting $v_{L} d p_{G}$ term is what has been done to obtain the eq's (1), (2) and the analogous equations in [4], because the gas phase there has been considered dilute. This is not our case, because we operate in the critical point vicinity, i.e. $v_{L}$ is comparable with $v_{G}$. Integrating the eq. (8)

$$
\int_{p_{s a t}}^{\tilde{p}_{s a t}}\left(v_{G}-v_{L}\right) d p_{G}=-v_{L} 2 \sigma / r
$$

we obtain approximate equation

$$
\left(v_{G}-v_{L}\right)\left(\tilde{p}_{s a t}-p_{s a t}\right) \approx-v_{L} 2 \sigma / r,
$$

which can be used if $v_{G}-v_{L}$ does not change significantly within interval $\left(\tilde{p}_{\text {sat }} ; p_{\text {sat }}\right)$.

Finally, we have

$$
\tilde{p}_{s a t}-p_{s a t} \approx-\frac{v_{L}}{v_{G}-v_{L}} 2 \sigma / r=-\frac{\rho_{G}}{\rho_{L}-\rho_{G}} 2 \sigma / r,
$$

where $\rho_{G}$ is the gas phase density.

Now we can rewrite eq. (8) in the following form

$$
(d \mu)_{T, n_{i}}=v_{L} d p_{L}=v_{G} d\left(p_{L}+2 \sigma / r\right) .
$$


Integrating it we obtain

$$
\int_{p_{s a t}}^{\tilde{p}_{\text {sat }}^{L}}\left(v_{L}-v_{G}\right) d p_{L}=v_{G} 2 \sigma / r,
$$

where $\tilde{p}_{\text {sat }}^{L}$ is the pressure of the liquid phase in equilibrium with the saturated vapor (both divided by the curved meniscus).

$$
\left(v_{L}-v_{G}\right)\left(\tilde{p}_{\text {sat }}^{L}-p_{\text {sat }}\right) \approx v_{G} 2 \sigma / r,
$$

Now we can write

$$
\tilde{p}_{s a t}^{L}-p_{s a t} \approx-\frac{v_{G}}{v_{G}-v_{L}} 2 \sigma / r=-\frac{\rho_{L}}{\rho_{L}-\rho_{G}} 2 \sigma / r .
$$

\section{Conclusions}

The eq. (11) (along with the eq. (14) and the eq's (9), (12) in the integral form) is the new form of the Kelvin equation that applies near the gas-liquid transition critical point. It can be helpful in researches of nano-structured materials. It should be applied carefully, due to the geometry dependence of the surface tension.

\section{References}

1. Thomson W. On the Equilibrium of Vapour at a Curved Surface of Liquid, Proc. Roy. Soc. Edinburgh, 7 (1870) 63-68.

2. Thomson W. On the Equilibrium of Vapour at a Curved Surface of Liquid, Philosophical Magazine Series 4, 42 (282) (1871) 448-452.

3. Gregg S.J., Sing K.S.W., Adsorption, Surface Science and Porosity, Academic Press, New York, 1982.

4. Parry A.O., Evans R. Universal fluctuation-induced corrections to the Kelvin equation for capillary condensation, J. Phys. A: Math. Gen. 25 (1992) 275-284. 